\title{
High Resolution Melt analysis for mutation screening in PKD1 and PKD2
}

Stanislas Bataille ${ }^{1,2}$, Yvon Berland ${ }^{2}$, Michel Fontes ${ }^{1}$ and Stéphane Burtey ${ }^{1,2^{*}}$

\begin{abstract}
Background: Autosomal dominant polycystic kidney disease (ADPKD) is the most common hereditary kidney disorder. It is characterized by focal development and progressive enlargement of renal cysts leading to end-stage renal disease. PKD1 and PKD2 have been implicated in ADPKD pathogenesis but genetic features and the size of PKD1 make genetic diagnosis tedious.

Methods: We aim to prove that high resolution melt analysis (HRM), a recent technique in molecular biology, can facilitate molecular diagnosis of ADPKD. We screened for mutations in PKD1 and PKD2 with HRM in 37 unrelated patients with ADPKD.

Results: We identified 440 sequence variants in the 37 patients. One hundred and thirty eight were different. We found 28 pathogenic mutations (25 in PKD1 and 3 in PKD2) within 28 different patients, which is a diagnosis rate of $75 \%$ consistent with literature mean direct sequencing diagnosis rate. We describe 52 new sequence variants in PKD1 and two in PKD2.

Conclusion: HRM analysis is a sensitive and specific method for molecular diagnosis of ADPKD. HRM analysis is also costless and time sparing. Thus, this method is efficient and might be used for mutation pre-screening in ADPKD genes.
\end{abstract}

\section{Background}

Autosomal dominant polycystic kidney disease (ADPKD) is a hereditary kidney disorder affecting approximately one in 500 to one in 1000 human live births [1]. It is characterized by focal development and progressive enlargement of renal cysts, leading to end-stage renal disease (ESRD) [2].

ADPKD is genetically heterogeneous and involves two genes, PKD1 (MIM 601313, chromosome region 16p13.3) [3] and PKD2 (MIM 173910, 4q21-22) [4]. Mutations in PKD1 account for approximately $85 \%$ of ADPKD cases and are associated with a more severe disease than PKD2 . The median age at onset of ESRD is 54.3 [52.7-55.9] years for individuals with mutation in PKD1 compared to 74.0 [67.2-80.8] years in PKD2 [5]. It has been hypothesized that a third gene could be implicated in ADPKD [6] but there is no evidence for this.

Genetic analysis of ADPKD is difficult owing to the existence of at least two distinct genes that can cause

\footnotetext{
* Correspondence: stephane.burtey@univmed.fr

'EA 4263 Thérapie des maladies génétiques, Faculté de médecine, Université de la méditerranée, Boulevard Jean Moulin 13005 Marseille, France

Full list of author information is available at the end of the article
}

disease and the lack of an exhaustive list of PKD1 and $P K D 2$ mutations that are associated with it. Genomic features of $P K D 1$ also cause difficulty in identifying sequence variants [7]. The open reading frame of $P K D 1$ is approximately $13 \mathrm{~kb}$ split in 46 exons. Exons 1-33 are duplicated six times at the $H G$ locus on a proximal position on chromosome 16p. The homology between the 5' region of $P K D 1$ and these six pseudo genes ranges from 94.5 to $96.7 \%$ in the duplicated area and almost $100 \%$ for the coding sequence [8]. Locus-specific amplifications of $P K D 1$ are required for analysis which complicates genetic diagnosis [9]. Furthermore, $P K D 1$ is a highly polymorphic gene with numerous sequence variants $[10,11]$ (Human Gene Mutation Database [HGMD]; http://www.hgmd.cf. ac.uk/ac/index.php[12]). Single nucleotide variants in the coding regions of the PKD genes could result in the development of ADPKD. Therefore, a strategy has been proposed to investigate the pathogenic significance of sequence variants in PKD1 [11].

Definitive diagnosis of ADPKD is based on an age-specific cystic renal phenotype and a positive family history [13]. Diagnosis of ADPKD in younger patients can be
Ciomed Central

() 2011 Bataille et al; licensee BioMed Central Ltd. This is an Open Access article distributed under the terms of the Creative Commons Attribution License (http://creativecommons.org/licenses/by/2.0), which permits unrestricted use, distribution, and reproduction in any medium, provided the original work is properly cited. 
difficult as renal ultrasonography can be inconclusive and if the family history is unknown. Molecular diagnosis could be of use in providing a definitive diagnosis.

High Resolution Melt (HRM) analysis, a recent advance in molecular biology used to detect variants in DNA sequences [14], has replaced $\mathrm{dHPLC}$, the reference screening method, for analyzing genetic variants of BRCA1 and BRCA2 [15]. There are major drawbacks with dHPLC including chemical waste, high maintenance costs and the need for post-PCR manipulations. Moreover, dHPLC does not allow high-throughput sequence variation screening.

HRM is based on the analysis of the melting curve of a real-time PCR amplicon. Monitoring the melting curve of the amplicon involves detecting the change in fluorescence intensity induced by the release of an intercalating DNA dye from a DNA duplex as it denatures at high temperatures. Unlike dHPLC, this technique allows high throughput screening. Even if the sequence is the endpoint of molecular analysis, a pre-screening step always reduces the cost compared to the whole gene sequencing [16]. Therefore, with regard to PKD1 and PKD2, HRM could be a rapid and cost effective technology for routine genetic analysis.

We show the results of an HRM screening strategy to identify sequence variants within PKD1 and PKD2 in 37 ADPKD patients and demonstrate that HRM is accurate to study ADPKD genes.

\section{Methods \\ DNA samples}

Thirty-seven unrelated patients from the Nephrology center of Marseille were screened for sequence variants in PKD1 and PKD2. They all fulfilled the ADPKD unified diagnosis criteria [17]. The cohort is composed of 14 females and 23 males. The mean age is 51 years $+/-11$. They had normal renal function ( 2 patients) or chronic renal failure (6 patients) or end-stage renal disease (29 patients). Fifty healthy individuals are the control group. They are from the same area and ethnic group as patients. Genomic DNA was extracted from lymphocytes harvested by venous blood puncture after written informed consent. Quantification and quality of all experimental DNA samples were assessed using Nanodrop ${ }^{\circledR}$ Technology (Coleman Technologies, Orlando, FL). DNA working solutions were diluted in ultrapure water to obtain the correct dilution. The study was conducted in compliance with the Helsinki Declaration and was approved by the Comite de Protection des Personnes (CPP) Sud-Méditerranée 2.

\section{Long Range PCR}

PKD1 screening previously required amplification of five specific long range (LR) PCR with primers designed in the PKD1 sequence differing from the homologues; this step ensures to screen for sequence variants in $P K D 1$, not in homologues. Long range PCR assays were performed as described previously [18], LR1 amplified exon 1, LR2 amplified exon 2 to 12, LR3 amplified exon 13 to 15D, LR4 amplified exon 15E to 22, LR5 amplified exon 22 to 32 . A sixth 7,838 bp LR PCR was designed to make HRM analysis for PKD1 exons 36 to 46 more specific with the forward and reverse primers: 5'-ACCTTCCCTC 'TAGGGAGGGAGCA-3' and 3'-GGCCAAGCTCGCATCCAAGCA-5'. Amplification was performed using the GeneAmp High Fidelity PCR System (Applied Biosystems, Foster City, CA) in a final volume of $50 \mu$ l containing $100 \mathrm{ng}$ gDNA, $2.5 \mathrm{U}$ enzyme, $15 \mu \mathrm{M}$ of each primer, $5 \mathrm{mM}$ dNTP, $5 \mu \mathrm{l}$ manufacturer's supplied buffer, $2 \mu \mathrm{l}$ supplied $\mathrm{MgCl}_{2}$ and $10 \%$ DMSO. Cycling conditions were $95^{\circ} \mathrm{C}$ for $3 \mathrm{~min}$, followed by 34 cycles at $96^{\circ} \mathrm{C}$ for $30 \mathrm{~s}, 62^{\circ} \mathrm{C}$ for $30 \mathrm{~s}, 68^{\circ} \mathrm{C}$ for $8 \mathrm{~min}$, and a last step of $7 \mathrm{~min}$ at $72^{\circ} \mathrm{C}$. All LR PCR products were verified on ethidium bromide stained gels.

\section{Primers}

We used oligonucleotide primers described in the literature to perform PCR on each PKD1 and PKD2 exons and on exon-intron junctions, which is important in order to identify variants affecting mRNA splicing. Primers from Tan et al. [18] and from Rossetti et al. [10] were used for PKD1 analysis; primers from Tan et al. [18] and from Hayashi et al. [19] were used for PKD2 . Forty-three out of 46 exons of $P K D 1$ were screened in 56 amplicons; 14 out of 15 exons of PKD2 were screened in 14 amplicons. Exons 1, 42, 43 of PKD1 and exon 1 of PKD2 could not be screened by HRM and were sequenced. Details of all primers are available in additional file 1.

\section{Real Time PCR and HRM conditions}

Amplification and HRM were performed in $20 \mu \mathrm{l}$ volumes in a LightCycler 480 (Roche Applied Systems). The amplification mixture included $20 \mathrm{ng}$ of genomic DNA or $5 \mu$ l of $1 / 500$ diluted PCR long range amplification product as template, $1 \mu \mathrm{l}$ of LightCycler 480 ResoLight Dye (Roche, Indianapolis, IN), $10 \mu \mathrm{L}$ of LightCycler 480 Probes Master (Roche, Indianapolis, IN) and $5 \mu \mathrm{M}$ of each primer.

Each run included an activation step at $95^{\circ} \mathrm{C}$ for $10 \mathrm{~min}$ followed by 45 amplification cycles of $15 \mathrm{~s}$ denaturation, $15 \mathrm{~s}$ annealing and $15 \mathrm{~s}$, elongation at $72^{\circ} \mathrm{C}$. Denaturation and annealing temperatures differed according to each fragment these are detailed in additional file 1.

First, the products were heated to $96^{\circ} \mathrm{C}$ for $1 \mathrm{~min}$ and temperature sample was reduced at $40^{\circ} \mathrm{C}$ for $1 \mathrm{~min}$. HRM was carried out with the temperature rising at a rate of $1^{\circ} \mathrm{C}$ per second with 25 acquisitions per degree. Initial and final temperatures also differed among amplicons (additional file 1). All reactions were performed in 96-well 
microtiter plates. Each fragment was tested in 10 patients per run.

\section{HRM analysis}

For HRM analysis, the melting curves were normalized and the temperature shifted (temp-shifted) so that samples were comparable. Modified curves were obtained with LC480 software in the gene-scanning module (version 1.3; Roche). The normalized and temp-shifted melting curves corresponded to the final curve after normalization. When an amplicon harbored a sequence variation, the normalized and temp-shifted melting curve had a different shape from wild-type amplicons.

In this study, normalization was manually adjusted; in a routine framework, normalization ranges are automatically filled out. Default software sensitivity setting was 30\% in order to avoid false negative amplicons. Therefore, sensitivity was set by default to $40 \%$ for all amplicons.

Comparing several wild-type amplicons demonstrated that the derived normalized and temp-shifted difference plot varies within a range owing to small random differences. The variance is partly caused by the specificity of the primers. Every amplicon that had a different derived normalized temp-shifted curve from wild-type was sequenced.

\section{Reverse transcription analysis}

To confirm the signification of the suspected splice mutation c.7210-5C>G, we performed RT-PCR from RNA extracted of leucocytes as previously described [20], briefly lymphocytes were isolated using a Ficoll gradient and total RNA was extracted using the Rneasy Qiagen kit, according to the manufacturer's instructions (Qiagen SA). Total RNA was resuspended in Rnase free water. A total of $5 \mu \mathrm{g}$ of total RNA was reverse transcribed using a RT-PCR kit (RT Life Technology GIBCO BRL), according to the manufacturer's instructions, and $3 \mu \mathrm{l}$ of the RT products were amplified using a standard hot start PCR technique using the primers, for PKD1 specific PCR (6F 5'-AGCGCAACTACTTGGAGGCCC-3' and 6R 5'-ACCACAACGGAG TTGGCGG-3') and we diluted this RT-PCR product to $1 / 1000$ and performed a nested PCR with the following primers (SAB26F2 5'-CAGGCCAATGTGACGGTGG-3' and SAB26B3 5'-CAGTCGCATGCCTGCACTGC-3').

\section{Sequencing}

Sequencing was used to confirm and investigate variants. All sequencing analysis was subcontracted to Cogenics ${ }^{\circledR}$ (Cogenics, Meylan, France) and performed by Sanger sequencing method. Capillary sequencing is performed using Big Dye* Terminator chemistry and sequences are delineated with Applied Biosystems $3730 \times 1$ platforms (http://www.beckmangenomics.com/documents/services/ GS_Sanger_Sequencing.pdf). Samples were prepared by diluting $20 \mu \mathrm{l}$ PCR product in $30 \mu \mathrm{l}$ of ultrapure water. Primers were diluted to obtain a concentration of $2 \mathrm{mM}$ as recommended by the subcontractor.

\section{Sequence variation analysis and classification}

All sequences were compared to the following references sequences NCBI RefSeq PKD1 : NM_000296.2; and PKD2 : NM_000297.2. The standard nomenclature recommended by HGVS (http://www.hgvs.org/mutnomen) was used to number nucleotides and name mutations.

For previously described sequence variants, we reported the clinical significance assessed in The Polycystic Kidney Disease Mutation Database accessible at http://pkdb.mayo.edu[12]. Pathogenic potential of novel mutations was assessed as follows: - nonsense or frameshift variants leading to a STOP codon as well as intronic mutations altering mRNA sequence proved with RT-PCR were considered as definitely pathogenic mutations. intronic and synonymous variants that did not alter predicted splicing (http://www.fruitfly.org/seq_tools/splice. $\mathrm{html}$ ) were considered as polymorphisms, the others as indeterminate mutations. - substitutions were evaluated using the scoring method described by Rossetti et al [11] and with PolyPhen, a software for interspecies sequence variations examination (http://genetics.bwh.harvard.edu/ pph) [21]. When the two scores assessed a pathogenic variant the mutation was considered as probably pathogenic, when they assessed a non pathogenic variant it was considered as polymorphism. If the two tests were discordant, the variation was reported as indeterminate.

\section{Results}

Forty-three exons of PKD1 were analyzed using HRM in 56 fragments after PCR amplification. Of the 2,072 amplicons tested in the 37 patients, 302 (14.6\%) were suspected to have a sequence variants compared to the wild-type amplicons analyzed (Figure 1). Each of the 302 amplicons was sequenced and 244 had at least one sequence variants. A total of 410 sequence variants were detected in PKD1, corresponding to $11.1 \pm 7.7$ (mean \pm SD) per patient; 3 exons were studied by direct sequencing. 129 different sequence variants were found.

Fourteen exons of PKD2 were analyzed in 14 fragments after PCR amplification. Of the 518 amplicons tested, HRM analysis identified 8 with sequence variants and 4 were shown to have at least one sequence variant after sequencing. A total of 30 sequence variants were detected in $P K D 2$, one exon was studied by direct sequencing. 9 different sequence variants were found.

Table 1 and Figure 2 show definitely pathogenic and probably pathogenic mutations. 25 mutations in $P K D 1$ and 3 in PKD2 were identified in 28 patients. Of these 28 mutations, 9 were already described in literature. Of the 


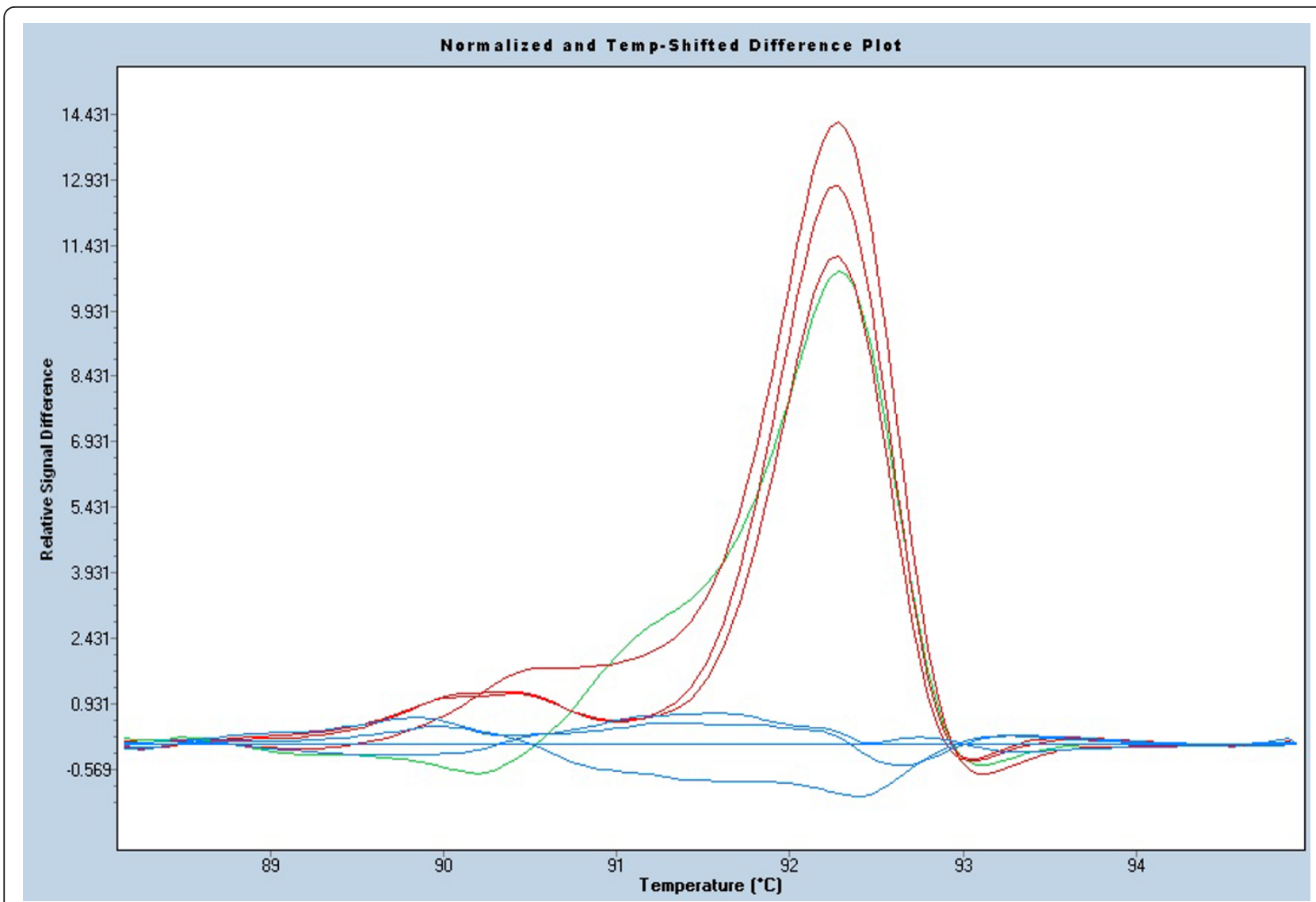

Figure 1 Example of HRM analysis result: normalized and temp-shifted result curve of fragment $15 \mathrm{D}$ of 10 patients. The three fragments with the red curves carry the same sequence variant p.Ala1555Ala (c.4665A>C), which is different from the fragment with the green curve p.Thr1558Thr (c.4674G>A). Fragments with the blue curve have no mutation.

19 new mutations, 13 were identified as being definitely pathogenic because they lead to a STOP codon; 3 substitutions were scored as probably pathogenic (Additional file 2) as well as 1 in frame deletions, one intronic deletion and one intronic substitution.

The first in frame deletion, p.Thr2337_Phe2338del, was classified as probably pathogenic because the two residues are highly conserved during evolution, they are located in the functional REJ domain and this deletion was the only probably pathogenic mutation identified in this patient (Figure 3). The second deletion was p. Leu2433del. Since leucine 2433 is a conserved residue (in chicken, frog and takifugu but not in mouse or rat), this variant was also considered probably pathogenic. It was the only possible mutation causing disease identified in this patient. In addition, this mutation was previously described associated with lack of expression of PC-1 in primary cilia [22]. The two in frame deletions were not found in 100 control chromosomes tested.

The 19 base pair intronic deletion in intron 31 (c.10167 +24del19) was considered as probably pathogenic. Unfortunately, this patient was lost of follow up and no RT-
PCR could be performed. A very similar deletion has previously been reported [23] as probably pathogenic in The Polycystic Kidney Disease Mutation Database. The deletion is present in three of the HG genes-PKD1 partial homologues on chromosome 16 (Figure 4) leading to a splice mutation associated with intron 31 retention. It was the only possible pathogenic mutation found in this patient and was not present in the 100 control chromosomes tested. The abnormal predicted splicing of intron 31 leads to a frameshift within exon 32 . The presence of this deletion in three of the six PKD1 homologues is of interest as it is associated with retention of the intron. A possible hypothesis for the pathogenesis of this mutation is the occurrence of recombination between homologue and PKD1. If a crossover event has occurred, it must be a small crossover, because the deletion region is flanked by two bases differing from the homologues and identical to PKD1 (Figure 4). The mutation c.7210-5C $>\mathrm{G}$ was predicted to alter splicing in silico and no other indeterminate or pathogenic variation was found in this patient. We classified this mutation as pathogenic after we performed a specific RT-PCR as previously described [20]. 
Table 1 Mutations

\begin{tabular}{|c|c|c|c|c|c|c|}
\hline DNA change & Protein change & Intron or exon & Fragment & Mutation type & Clinical significance & Reference \\
\hline \multicolumn{7}{|c|}{ PKD1 } \\
\hline c. $862 C>T$ & p.GIn288X & Exon 5 & $5 B$ & NONSENSE & Definitely pathogenic & Novel \\
\hline c.926_939del14 & p.Asp309fs & Exon 5 & $5 B$ & FRAMESHIFT & Definitely pathogenic & Novel \\
\hline c.2073G $>$ A & p.Ala692Thr & Exon 10 & 10 & SUBSTITUTION & Probably pathogenic & Novel \\
\hline c.3783_3784insC & p.Glu1261fs & Exon 15 & $15 B$ & FRAMESHIFT & Definitely pathogenic & Novel \\
\hline C.5078C $>$ A & p.Tyr1693X & Exon 15 & $15 \mathrm{E}$ & NONSENSE & Definitely pathogenic & Novel \\
\hline$c .5610 C>G$ & p.Asn1870Lys & Exon 15 & $15 \mathrm{~F}$ & SUBSTITUTION & Probably pathogenic & Novel \\
\hline C. $5923 C>T$ & p.GIn1975X & Exon 15 & $15 G$ & NONSENSE & Definitely pathogenic & Novel \\
\hline c.7008_7013delCCTTCA & p.Thr2337_Phe2338del & Exon 16 & 16 & DELETION & Probably pathogenic & Novel \\
\hline c. $7108 \mathrm{~T}>\mathrm{A}$ & p.Cys 2370 Ser & Exon 17 & 17 & SUBSTITUTION & Probably pathogenic & {$[11]$} \\
\hline c.7155_7156insT & p.Tyr2386fs & Exon 17 & 17 & FRAMESHIFT & Definitely pathogenic & Novel \\
\hline c.7298_7300delTGC & p.del2433Leu & Exon 18 & 18 & DELETION & Probably pathogenic & [22] \\
\hline c. $7210-5 C>G$ & pThr2496fs & Intron 18 & 19 & SPLICE & Definitely pathogenic & Novel \\
\hline c.8124_8127delGCCC & p.Thr2710fs & Exon 22 & 22 & FRAMESHIFT & Definitely pathogenic & Novel \\
\hline c.9147_9148insG & p.Ala3050fs & Exon 25 & 25 & FRAMESHIFT & Definitely pathogenic & Novel \\
\hline c. $9403 C>T$ & p.Thr3135Met & Exon 27 & 27 & SUBSTITUTION & Probably pathogenic & Novel \\
\hline c.9203_9205delGTG & p.del3138Val & Exon 27 & 27 & DELETION & Probably pathogenic & [23] \\
\hline c.9859_9861delCTC & p.del3287Leu & Exon 29 & 29 & DELETION & Probably pathogenic & {$[34]$} \\
\hline c. $10086 C>T$ & p.Gln3363X & Exon 31 & 31 & NONSENSE & Definitely pathogenic & Novel \\
\hline c. $10167+24$ del 19 & p.Gln3389fs & Intron 31 & 31 & SPLICE & Probably pathogenic & Novel \\
\hline c.10932delC & p.Arg3646fs & Exon 37 & 37 & FRAMESHIFT & Definitely pathogenic & Novel \\
\hline c.10945_10952del8 & p.3649fs & Exon 37 & 37 & FRAMESHIFT & Definitely pathogenic & Novel \\
\hline c. $11249 \mathrm{G}>\mathrm{A}$ & p.Arg3750Gln & Exon 39 & 39 & SUBSTITUTION & Probably pathogenic & {$[32]$} \\
\hline c. $11512 C>T$ & p.Gln3838X & Exon 41 & 41 & NONSENSE & Definitely pathogenic & {$[35]$} \\
\hline c.11972delC & p.Ala3991fs & Exon 43 & 43 & FRAMESHIFT & Definitely pathogenic & Novel \\
\hline \multirow[t]{2}{*}{ c. $12235+2 T>C$} & p.Leu4046fs & Exon 44 & 44 & SPLICE & Definitely pathogenic & [20] \\
\hline & & & PKD2 & & & \\
\hline c.640G $>T$ & p.Glu214X & Exon 2 & 2 & NONSENSE & Definitely pathogenic & Novel \\
\hline c. $974 \mathrm{G}>\mathrm{A}$ & p.Arg325Gln & Exon 4 & 4 & SUBSTITUTION & Probably pathogenic & [11] \\
\hline c. $2533 C>T$ & p.Arg845X & Exon 14 & 14 & NONSENSE & Definitely pathogenic & {$[36]$} \\
\hline
\end{tabular}

No mutation was detected in more than one individual.

The mutation is responsive of the retention of intron 18 leading to a frameshift with a stop codon in position 2514.

Polymorphisms are presented in Table 2. We identified 92 polymorphisms in PKD1 and 6 in PKD2. Two intronic variants in $P K D 1$ were predicted to alter splicing but were considered as polymorphisms, c. 2985 $+4 \mathrm{G}>\mathrm{A}$ was present in 4 patients, a definite pathogenic mutation was identified in three and c.2985+5G $>$ A was present in three individuals who already had a pathogenic mutation. The p.Glu107Asp novel substitution in PKD2 was classified polymorphism because it was found in 15 patients of the cohort, which is not consistent with a pathogenic mutation.

We found 12 indeterminate sequence variants in PKD1 and none in PKD2 . They are presented in Table 3. The majority of these variants were substitutions; their scoring is presented in Additional file 2. The small in frame insertion p.Asp3781_Val3782insGlu was considered as indeterminate because the patient had another indeterminate variant (p.Arg4276Trp) and we could not be sure of its pathogenicity. The three other indeterminate sequence variants (p.Ala2704Val, p.Ser2935Phe p. His3559Pro) are the sole mutation with potential pathogenicity observed in the three individuals screened.

\section{Discussion}

We describe the first sequence variants pre-screening method for PKD1 and PKD2 using HRM analysis. This is the first use of this technique in genetic nephrology. This method has a good diagnosis rate as evidenced by the identification of a mutation in $75 \%$ of our cohort (including definitely pathogenic and probably pathogenic mutations), which is the mean diagnosis rate reported with direct sequencing of the two genes [24]. In comparison, Rossetti et al found a diagnosis rate of $62.9 \%$ with direct sequencing [11] and 67\% with dHPLC [10]. In addition a recent report of Hoefele et al found a 


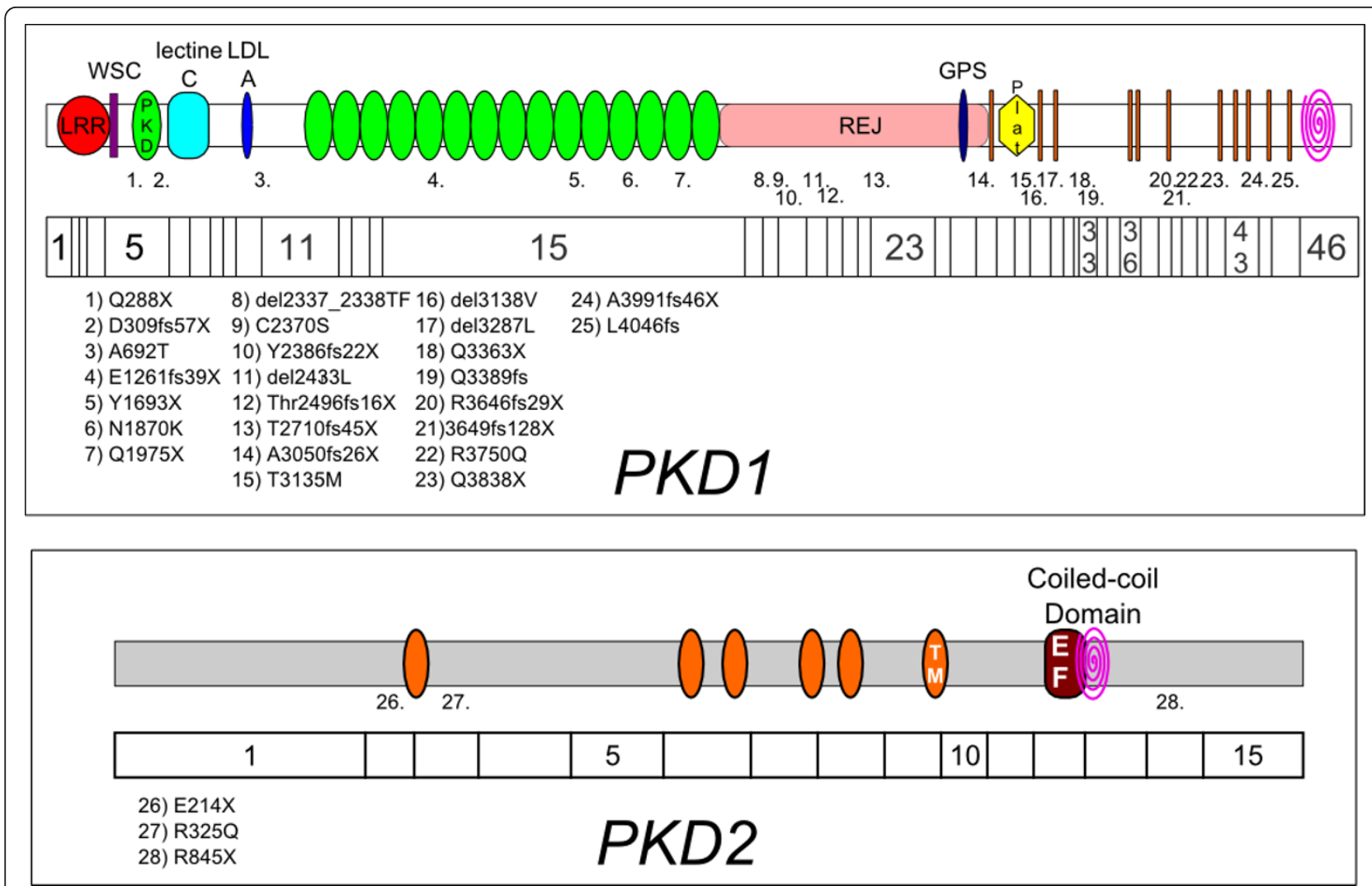

Figure 2 Pathogenic mutations in PKD1 and PKD2. The pathogenic variants of PKD1 and PKD2 are positioned in a representation of genes and proteins.

mutation detection efficiency of $64,5 \%$ by direct sequencing [25].

More than $81 \%$ of amplicons suspected to have a sequence variant after HRM analysis carried one after sequencing, confirming the specificity of this technology. Overall, less than $15 \%$ of the fragments of PKD1 and $1.5 \%$ of the PKD2 fragments analyzed by HRM were sequenced. Moreover, we screened in a blind procedure four patients with previously known mutations [20] and identified all of them. Thus, HRM is efficient, sensitive and specific for mutation screening in ADPKD genes. HRM analysis reduced drastically the need for systematic sequencing and the time of sequence analysis.

We describe 52 new sequence variants in PKD1: 18 were classified as mutation, no mutation was detected in more than one individual, 23 as polymorphism and 11 as indeterminate variant. We report one new mutation and one polymorphism in PKD2 .

Large deletions or duplications represent $4 \%$ of ADPKD pathogenic variants [26]. HRM technology allows genomic qPCR screening to be performed for large deletions/duplications in the same run. Successful simultaneous analysis by HRM and genomic qPCR has been described for $M L H 1$ gene [27]. Therefore, it is likely that HRM and genomic qPCR can be performed simultaneously for $P K D 1$ and $P K D 2$, which will require the introduction of control DNA and a reference gene in the microtiter plate. This will improve molecular diagnosis by directly identifying large deletions/duplications that have not been identified by HRM analysis or with sequencing. Therefore, HRM analysis could prove to be a highly integrated molecular diagnosis tool.

HRM analysis is a high throughput technique for sequence variants screening $P K D 1$ and $P K D 2$. The format of PCR analysis in 96 or 394 well plates allows the process to be automated after DNA extraction and the first round of LR PCR for PKD1. A major advantage of HRM is a drastic diminution in the needs for sequencing to identify a sequence variation. HRM could be more cost effective than direct sequencing for identification of molecular anomalies in ADPKD. Comparing to dHPLC, HRM is a closed tube and a non destructive technique. The PCR and analytical steps are in the same run with HRM. During dHPLC several separate steps require PCR products manipulation and can induce mistakes. After analysis, in HRM the PCR product can be directly sequenced; in dHPLC the PCR product is destroyed during the run and a new PCR must be done for sequencing. 


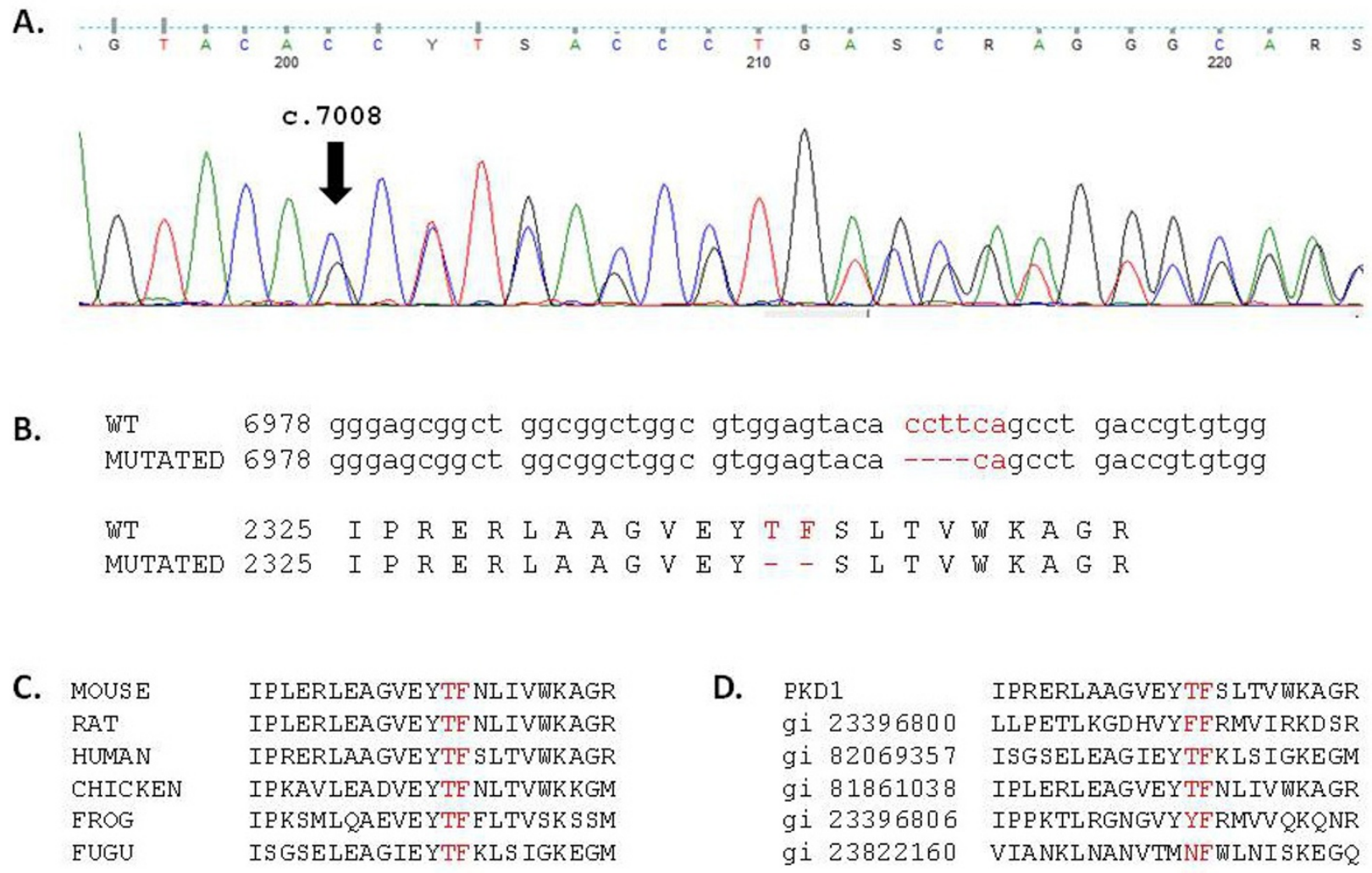

Figure 3 Pathogenic variant p. Thr2337_Phe2338del. A. Electrophoregram centered on sequence variant. The black arrow indicates the first deleted base. B. Alignments between wild type (WT) and mutated DNA sequences (top) and wild-type and mutated protein sequence (bottom). C. Alignments between deleted region and orthologs. D. Alignments between deleted region and conserved REJ domains.

In our facility, the price for one amplicon analysis with HRM is 1.52 \$ (1.10 €) compared to 4.64 \$ (3.36 €) with dHPLC [28]. In addition, HRM allows large fragment rearrangements detection in the same step. This technique is very competitive compared to dHPLC. HRM is time sparing, provides greater security (closed tube during the whole process) and is cheaper. Assuming bidirectional sequencing costs $13.8 \$(10 €)$ per fragment, the cost for mutation screening in PKD1 and PKD2 with direct sequencing costs $966 \$(700 €)$ (70 fragments). With HRM, 70 fragments $\times 1.52 \$$ plus sequencing of positive fragments (i.e. 9 fragments $\times 13.8 \$$ ), the total cost is of $230.46 \$(167 €)$. Furthermore, we do not consider here the technician time sparing provided by HRM in this cost analysis. Thus, acquiring the needed hardware for HRM analysis is easily paid off. HRM pre-screening is cost and time efficient facing direct sequencing in ADPKD genes.

The technique efficiency will soon be further improved with the search of conditions allowing the screening of exons 1 of PKD1 and PKD2 and exons 42 and 43 of PKD1 . We were not able to conduct HRM analysis of these exons because the denaturation curves could not be analyzed. We suspected this failure is related to the high GC contents of the DNA sequence of exon 1 of $P K D 1$ and $P K D 2$. For exon 42 and 43 of $P K D 1$, we suspected the formation of secondary structures not solved by denaturation temperature. HRM is a rather young technology and we can expect important developments increasing the efficiency of this sensitive and specific method of screening [28].

Molecular diagnosis of ADPKD is important for improving the care of patients [29]. Renal transplantation from a related living donor at risk of ADPKD is a concern since clinical and ultrasonographic diagnosis criteria fail to identify donors with ADPKD when they are aged less than 40 years [30]. Molecular diagnosis could be of use in this area as well as identifying mutations in ambiguous phenotypes [31], for example when the family history is unknown. Genetic diagnosis also results in a more accurate prognosis [32] as the genes mutated in ADPKD are the prognostic factors linked to kidney survival [33]. In addition, therapies development will probably require an accurate diagnosis in young 
A.
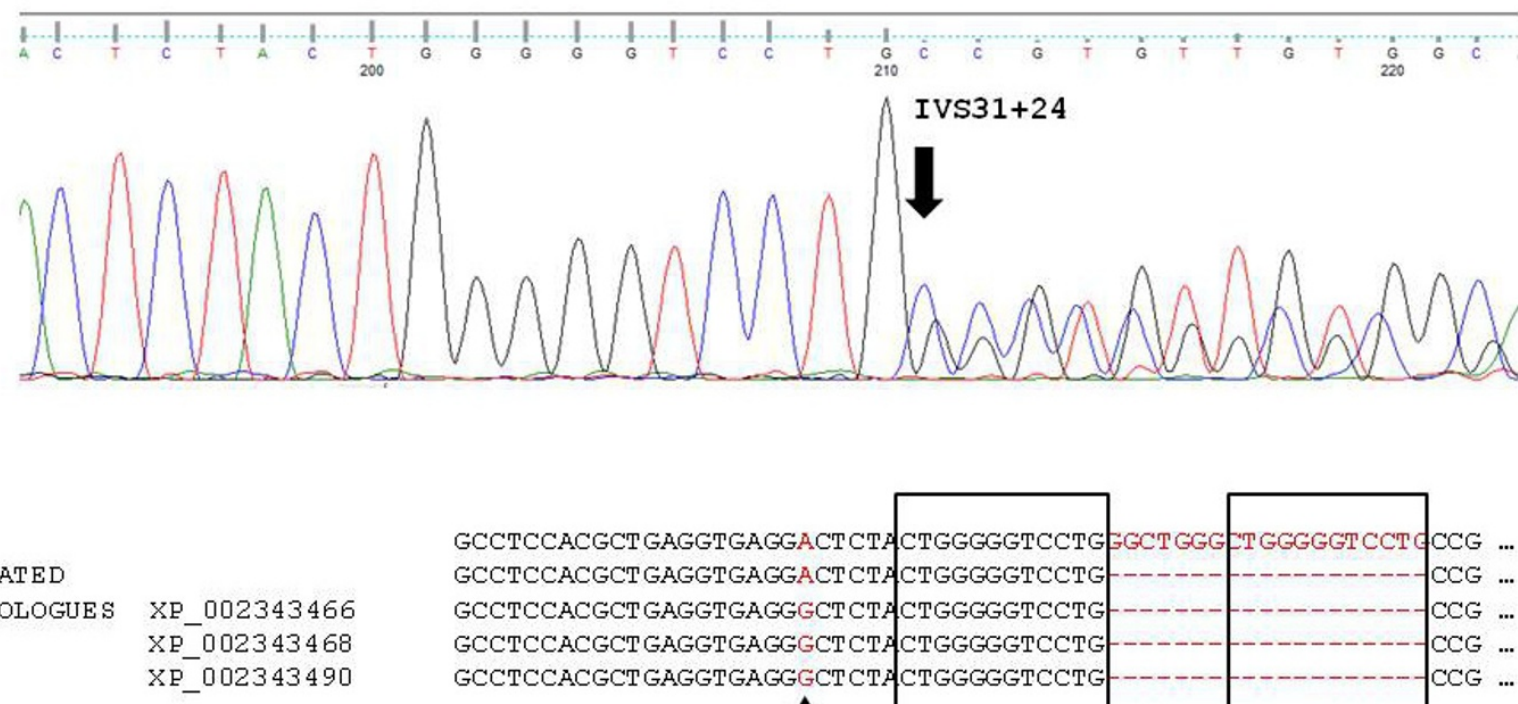

\section{IVS $31+24$}

B.

\section{WT}

MUTATED
HOMOLOGUES XP_002343490

WT

MUTATED

HOMOLOGUES
XP_002343466

XP_0023434668

XP_002343490

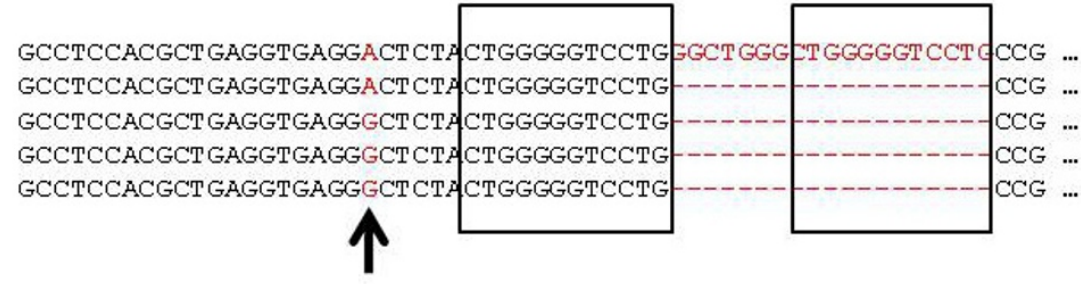

... CCTTGGCGCAGCTTGGACT CAAGACACT GT GCACCT CTCAGCAGGCCTTTGTTGGACAGA

... CCTT GGCGCAGCT TGGACT CAAGACACT GT GCACCT CTCAGCAGGCCTTTGTTGGACAGA.

... CCTTGGCGCAGCTTGGACTCA.AGACCCTGTGCACCT CTCAGCAGGCCTTTGCTGGACAGA.

... CCTTGGCGCAGCTTGGACT CAAGACCCT GT GCACCT CTCAGCAGGCCTTTGCTGGACAGA.

... CCTTGGCGCAGCTTGGACT CA.AGACCCT GT GCACCT CTCAGCAGGCCTTTGCTGGACAGA.

Figure 4 Mutation. c.10167+24del19. A. Electrophoregram centered on sequence variant. The black arrow indicates the location of the deletion. B. Alignments between wild type (WT), mutated and homologues DNA sequences. Black arrows show the flanking bases in the mutated sequence that is identical to WT but different from the homologues sequence. Black boxes represent a repeated 12 bp regions.

Table 2 Polymorphisms

\begin{tabular}{|c|c|c|c|c|c|c|}
\hline DNA change & Protein change & Intron or exon & Fragment & Mutation type & Reference & $\mathrm{Nb}$ \\
\hline \multicolumn{7}{|c|}{ PKD1 } \\
\hline c.107C>A & p.Pro36His & Exon 1 & 1 & SUBSTITUTION & {$[37]$} & 2 \\
\hline c. $114 C>T$ & p.Leu38Leu & Exon 1 & 1 & SYNONYMOUS & Novel & 1 \\
\hline c. $1023 C>T$ & p.Ala341Ala & Exon 5 & $5 C$ & SYNONYMOUS & [9] & 2 \\
\hline C.1119T>C & p.Leu373Leu & Exon 5 & $5 C$ & SYNONYMOUS & [24] & 1 \\
\hline c.1323G >A & p.Gly441Gly & Exon 6 & 6 & SYNONYMOUS & {$[25]$} & 1 \\
\hline c. $1607-27 C>T$ & Silent & Intron 7 & 8 & INTRONIC & [10] & 3 \\
\hline C.1714C>T & p.Pro572Ser & Exon 8 & 8 & SUBSTITUTION & [10] & 1 \\
\hline c. $1850-4 A>G$ & Silent & Intron 9 & 10 & INTRONIC & [10] & 2 \\
\hline c. $2214 C>G$ & p.Pro738Pro & Exon 11 & $11 \mathrm{~A}$ & SYNONYMOUS & [18] & 1 \\
\hline c. $2216 A>G$ & p.GIn739Arg & Exon 11 & $11 \mathrm{~A}$ & SUBSTITUTION & [31] & 5 \\
\hline c. $2234 C>G$ & p.Ala745Ala & Exon 11 & $11 \mathrm{~A}$ & SYNONYMOUS & Novel & 1 \\
\hline c. $2700 \mathrm{G}>\mathrm{A}$ & p.Pro900Pro & Exon 11 & $11 \mathrm{~B}$ & SYNONYMOUS & {$[31]$} & 1 \\
\hline$c .2730 C>T$ & p.Asp910Asp & Exon 11 & $11 \mathrm{~B}$ & SYNONYMOUS & {$[31]$} & 1 \\
\hline c. $2854-5 C>T$ & Silent & Intron 11 & 12 & INTRONIC & {$[10]$} & 1 \\
\hline c. $2985+4 G>A$ & Silent & Intron 12 & 12 & INTRONIC & Novel & 4 \\
\hline c. $2985+5 G>A$ & Silent & Intron 12 & 12 & INTRONIC & Novel & 3 \\
\hline c. $2986-15 C>T$ & Silent & Intron 12 & 13 & INTRONIC & [38] & 4 \\
\hline c.3063T>C & p.Gly1021Gly & Exon 13 & 13 & SYNONYMOUS & {$[31]$} & 8 \\
\hline c. $3111 A>G$ & p.Leu1037Leu & Exon 13 & 13 & SYNONYMOUS & {$[9]$} & 7 \\
\hline
\end{tabular}


Table 2 Polymorphisms (Continued)

\begin{tabular}{|c|c|c|c|c|c|c|}
\hline c.3275T>C & p.Met1092Thr & Exon 14 & 14 & SUBSTITUTION & {$[10]$} & 5 \\
\hline c. $2395+53 G>T$ & Silent & Intron 14 & 14 & INTRONIC & Novel & 1 \\
\hline c. $3372 C>T$ & p.Ala1124Ala & Exon 15 & $15 \mathrm{~A}$ & SYNONYMOUS & [9] & 8 \\
\hline c.3375C>T & p.Ser1125Ser & Exon 15 & $15 \mathrm{~A}$ & SYNONYMOUS & [9] & 8 \\
\hline c. $3502 C>T$ & p.Pro1168Ser & Exon 15 & $15 \mathrm{~A}$ & SUBSTITUTION & [10] & 2 \\
\hline$c .4018 C>T$ & p.Arg1340Trp & Exon 15 & $15 B$ & SUBSTITUTION & [10] & 1 \\
\hline$c .4071 \mathrm{G}>\mathrm{T}$ & p.Leu1357Leu & Exon 15 & $15 C$ & SYNONYMOUS & [18] & 1 \\
\hline c.4195T>C & p.Trp1399Arg & Exon 15 & $15 C$ & SUBSTITUTION & [31] & 8 \\
\hline c. $4546 G>A$ & p.Ala1516Thr & Exon 15 & $15 D$ & SUBSTITUTION & [18] & 1 \\
\hline c. $4665 \mathrm{~A}>\mathrm{C}$ & p.Ala1555Ala & Exon 15 & $15 D$ & SYNONYMOUS & [31] & 11 \\
\hline c. $4674 G>A$ & p.Thr1558Thr & Exon 15 & $15 D$ & SYNONYMOUS & [38] & 4 \\
\hline$c .5051 C>T$ & p.Ser1684Leu & Exon 15 & $15 \mathrm{E}$ & SUBSTITUTION & [10] & 1 \\
\hline $\mathrm{C} .5172 \mathrm{C}>\mathrm{T}$ & p.Ala1724Ala & Exon 15 & $15 \mathrm{E}$ & SYNONYMOUS & [39] & 11 \\
\hline c. $5763 G>A$ & p.Leu1921Leu & Exon 15 & $15 G$ & SYNONYMOUS & [9] & 1 \\
\hline c.6927C>T & p.Gly2309Gly & Exon 16 & 16 & SYNONYMOUS & [39] & 2 \\
\hline C. $7165 T>C$ & p.Leu2389Leu & Exon 17 & 17 & SYNONYMOUS & {$[31]$} & 7 \\
\hline c. $7209+16 \mathrm{G}>\mathrm{A}$ & Silent & Intron 17 & 17 & INTRONIC & Novel & 13 \\
\hline c. $7210 C>T$ & p.Arg2404Trp & Exon 18 & 18 & SUBSTITUTION & CRISP unpublised & 1 \\
\hline c. $7441 C>T$ & p.Leu2481Leu & Exon 18 & 18 & SYNONYMOUS & {$[31]$} & 11 \\
\hline c.7642G>C & p.Glu2548Gln & Exon 19 & 19 & SUBSTITUTION & [39] & 1 \\
\hline c. $7703+24 C>A$ & Silent & Intron 19 & 19 & INTRONIC & [18] & 1 \\
\hline C.7708T>C & p.Leu2570Leu & Exon 20 & 20 & SYNONYMOUS & [32] & 8 \\
\hline c. $7863+47 \mathrm{~T}>\mathrm{G}$ & Silent & Intron 20 & 20 & INTRONIC & [10] & 9 \\
\hline c.7913A>G & p.His2638Arg & Exon 21 & 21 & SUBSTITUTION & {$[38]$} & 8 \\
\hline c. $8016+13$ delG & Silent & Intron 21 & 21 & INTRONIC & Novel & 1 \\
\hline c. $8016+26 \mathrm{~T}>\mathrm{C}$ & Silent & Intron 21 & 21 & INTRONIC & {$[30]$} & 8 \\
\hline c. $8016+71 G>A$ & Silent & Intron 21 & 21 & INTRONIC & Novel & 1 \\
\hline c. $8016+77 C>T$ & Silent & Intron 21 & 21 & INTRONIC & Novel & 1 \\
\hline C.8020C $>T$ & p.Pro2674ser & Exon 22 & 22 & SUBSTITUTION & {$[40]$} & 2 \\
\hline $\mathrm{c} .8087 \mathrm{~T}>\mathrm{G}$ & p.Leu2696Arg & Exon 22 & 22 & SUBSTITUTION & [41] & 12 \\
\hline $\mathrm{c} .8123 \mathrm{C}>\mathrm{T}$ & p.Thr2708Met & Exon 22 & 22 & SUBSTITUTION & {$[40]$} & 1 \\
\hline c. $8161+21 \mathrm{~T}>\mathrm{C}$ & Silent & Intron 22 & 22 & INTRONIC & {$[30]$} & 13 \\
\hline$c .8161+23 C>T$ & Silent & Intron 22 & 22 & INTRONIC & Novel & 9 \\
\hline c. $8161+24 C>G$ & Silent & Intron 22 & 22 & INTRONIC & Novel & 11 \\
\hline$c .8161+25 A>G$ & Silent & Intron 22 & 22 & INTRONIC & Novel & 11 \\
\hline c.8161+29del18 & Silent & Intron 22 & 22 & INTRONIC & Novel & 1 \\
\hline c. $8161+30 C>G$ & Silent & Intron 22 & 22 & INTRONIC & Novel & 12 \\
\hline$C .8161+31 C>T$ & Silent & Intron 22 & 22 & INTRONIC & Novel & 12 \\
\hline $\mathrm{c} .8161+38 \mathrm{G}>\mathrm{A}$ & Silent & Intron 22 & 22 & INTRONIC & {$[30]$} & 10 \\
\hline C. $8161+39 \mathrm{~T}>\mathrm{C}$ & Silent & Intron 22 & 22 & INTRONIC & {$[10]$} & 1 \\
\hline C. $8161+41 C>T$ & Silent & Intron 22 & 22 & INTRONIC & Novel & 1 \\
\hline C. $8161+42 C>G$ & Silent & Intron 22 & 22 & INTRONIC & Novel & 10 \\
\hline c. $8161+46$ ins 18 & Silent & Intron 22 & 22 & INTRONIC & Novel & 1 \\
\hline c. $8644 \mathrm{~T}>\mathrm{A}$ & p.Trp2882Arg & Exon 23 & $23 B$ & SUBSTITUTION & {$[18]$} & 1 \\
\hline c.8681_8689del9 & p.Ala2894_Ser2896del & Exon 23 & $23 B$ & DELETION & [11] & 1 \\
\hline C.8898G $>C$ & p.Glu2966Asp & Exon 24 & 24 & SUBSTITUTION & [42] & 1 \\
\hline C.8913T>C & p.Ala2971Ala & Exon 24 & 24 & SYNONYMOUS & [42] & 2 \\
\hline c. $8948+17 A>G$ & Silent & Intron 24 & 24 & INTRONIC & [18] & 1 \\
\hline C.9195G>C & p.Val3065Val & Exon 25 & 25 & SYNONYMOUS & Novel & 1 \\
\hline C.9196T>C & p.Phe3066Leu & Exon 25 & 25 & SUBSTITUTION & {$[43]$} & 1 \\
\hline c. $9260 C>G$ & p.Thr3087Thr & Exon 26 & 26 & SYNONYMOUS & Novel & 1 \\
\hline$c .9270 C>T$ & p.Val3090Val & Exon 26 & 26 & SYNONYMOUS & [39] & 2 \\
\hline
\end{tabular}


Table 2 Polymorphisms (Continued)

\begin{tabular}{|c|c|c|c|c|c|c|}
\hline C.9330T>C & p.Pro3110Pro & Exon 26 & 26 & SYNONYMOUS & [44] & 12 \\
\hline c.9569-13T>C & Silent & Intron 27 & 28 & INTRONIC & {$[10]$} & 5 \\
\hline c. $9712+30 \mathrm{~T}>\mathrm{G}$ & Silent & Intron 28 & 28 & INTRONIC & Novel & 2 \\
\hline c. $10050+54 A>G$ & Silent & Exon 30 & 30 & INTRONIC & {$[10]$} & 5 \\
\hline c. $10170+14 T>C$ & Silent & Intron 31 & 31 & INTRONIC & {$[45]$} & 1 \\
\hline C.10225G>C & p.Val3409Leu & Exon 33 & 33 & SUBSTITUTION & [18] & 1 \\
\hline c. $10368 C>T$ & p.Ala3456Ala & Exon 33 & 33 & SUBSTITUTION & {$[18]$} & 1 \\
\hline c. $10406-4 C>T$ & Silent & Intron 33 & 34 & INTRONIC & Novel & 1 \\
\hline c. $10535 C>T$ & p.Ala3512Val & Exon 35 & 35 & SUBSTITUTION & {$[44]$} & 4 \\
\hline c. $10768 C>T$ & p.Leu3590Leu & Exon 36 & 36 & SYNONYMOUS & {$[46]$} & 5 \\
\hline C.11376G >C & p.Ser3792Ser & Exon 40 & 40 & SYNONYMOUS & {$[40]$} & 1 \\
\hline c.11537+5_+6insGGG & Silent & Intron 41 & 41 & INTRONIC & [39] & 1 \\
\hline c. $11682 C>T$ & p.Ser3894Ser & Exon 42 & 42 & SYNONYMOUS & [10] & 2 \\
\hline c. $11916 C>T$ & p.Arg3972Arg & Exon 43 & 43 & SYNONYMOUS & [39] & 5 \\
\hline c. $12138+22$ delG & Silent & Intron 44 & 44 & INTRONIC & [47] & 1 \\
\hline C.12176C>T & p.Ala4059Val & Exon 45 & 45 & SUBSTITUTION & [48] & 3 \\
\hline c. $12201 T>C$ & p.Pro4067Pro & Exon 45 & 45 & SYNONYMOUS & Novel & 9 \\
\hline c. $12276 \mathrm{~A}>\mathrm{G}$ & p.Ala4092Ala & Exon 45 & 45 & SYNONYMOUS & {$[31]$} & 5 \\
\hline c. $12409 C>T$ & p.Leu4137Leu & Exon 45 & 45 & SYNONYMOUS & [49] & 1 \\
\hline c.12630T>C & p.Pro4210Pro & Exon 46 & $46 \mathrm{~A}$ & SYNONYMOUS & {$[46]$} & 1 \\
\hline c. $12765 C>T$ & p.Pro4255Pro & Exon 46 & $46 \mathrm{~B}$ & SYNONYMOUS & {$[50]$} & 1 \\
\hline \multicolumn{7}{|c|}{ PKD2 } \\
\hline c. $83 G>C$ & p.Arg28Pro & Exon 1 & $1 \mathrm{~A}$ & SUBSTITUTION & {$[51]$} & 1 \\
\hline c. $321 A>T$ & p.Glu107Asp & Exon 1 & $1 \mathrm{~B}$ & SUBSTITUTION & Novel & 15 \\
\hline c. $420 \mathrm{G}>\mathrm{A}$ & p.Gly140Gly & Exon 1 & $1 \mathrm{~B}$ & SYNONYMOUS & {$[52]$} & 6 \\
\hline C.568G $>A$ & p.Ala190Thr & Exon 1 & $1 \mathrm{~B}$ & SUBSTITUTION & {$[25]$} & 3 \\
\hline c. $844-22 \mathrm{G}>\mathrm{A}$ & Silent & Intron 3 & 4 & INTRONIC & [51] & 1 \\
\hline c.1445T>G & p.Phe482Cys & Exon 6 & 6 & SUBSTITUTION & [53] & 1 \\
\hline
\end{tabular}

$\mathrm{Nb}$ : number of patients carrying the sequence variant.

adults to initiate treatment before significant renal changes have occurred. HRM could be used as a quick and cost effective genetic test for individuals included in clinical trials.

\section{Conclusions}

HRM analysis is an efficient, sensitive, specific and cost effective strategy to identify mutations in PKD1 and PKD2 .

Table 3 Indeterminate variants

\begin{tabular}{|c|c|c|c|c|c|c|}
\hline DNA change & Protein change & Intron or exon & Fragment & Mutation type & Reference & $\mathrm{Nb}$ \\
\hline \multicolumn{7}{|c|}{ PKD1 } \\
\hline $\mathrm{c.854C}>\mathrm{T}$ & p.Ala285Val & Exon 5 & $5 B$ & SUBSTITUTION & Novel & 1 \\
\hline c.4439A>G & p.Glu1480Gly & Exon 15 & $15 C$ & SUBSTITUTION & Novel & 1 \\
\hline C. $5848 \mathrm{G}>\mathrm{A}$ & p.Val1950Met & Exon 15 & $15 G$ & SUBSTITUTION & Novel & 1 \\
\hline$c .8110 C>T$ & p.Ala2704Val & Exon 22 & 22 & SUBSTITUTION & Novel & 1 \\
\hline c. $8803 C>T$ & p.Ser2935Phe & Exon 24 & 24 & SUBSTITUTION & Novel & 1 \\
\hline$c .9730 G>A$ & p.Arg3244His & Exon 29 & 29 & SUBSTITUTION & Novel & 1 \\
\hline c. $10043 G>A$ & p.Arg3348Gln & Exon 30 & 30 & SUBSTITUTION & Novel & 1 \\
\hline c. $10876 A>C$ & p.His3559Pro & Exon 36 & 36 & SUBSTITUTION & Novel & 2 \\
\hline C.11108G>C & p.Ser3703Thr & Exon 38 & 38 & SUBSTITUTION & Novel & 4 \\
\hline c.11344_11345insAAG & p.Asp3781_Val 3782insGlu & Exon 40 & 40 & INSERTION & Novel & 1 \\
\hline c.11698C>T & p.Leu3897Phe & Exon 42 & 42 & SUBSTITUTION & Novel & 1 \\
\hline C. $12826 C>T$ & p.Arg4276Trp & Exon 46 & $46 B$ & SUBSTITUTION & {$[54]$} & 1 \\
\hline
\end{tabular}

$\mathrm{Nb}$ : number of patients carrying the sequence variant. 


\section{Additional material}

Additional file 1: Primers used for PKD1 and PKD2 analysis. The file contains the sequence and characteristic of the primers used for amplification of PKD1 and PKD2

Additional file 2: Scoring details of novel coding sequence variations. The file contains the score to evaluate the pathogenicity of the new sequence variations described in the text.

\section{Acknowledgements}

We thank P. Harris and S. Rossetti for providing the scoring scale for substitutions. This work was supported by a grant from the Fondation du Rein and a grant from BQR 2009 Université de la Méditerranée.

\section{Author details}

'EA 4263 Thérapie des maladies génétiques, Faculté de médecine, Université de la méditerranée, Boulevard Jean Moulin 13005 Marseille, France. ${ }^{2}$ Centre de néphrologie et transplantation rénale, Hôpital de la Conception, 147 Boulevard Baille 13005 Marseille, France.

\section{Authors' contributions}

SB carried out the molecular genetic studies, participated in the sequence alignment and drafted the manuscript. YB participated to recruitment of patients. MF participated in the design of the study and drafted the manuscript. SB conceived of the study, and participated in its design and coordination, and participated to recruitment of patients and helped to draft the manuscript. All authors read and approved the final manuscript.

\section{Competing interests}

All authors declared to have non-financial competing interests in relation to this manuscript.

Received: 22 January 2011 Accepted: 18 October 2011

Published: 18 October 2011

\section{References}

1. Levy M, Feingold J: Estimating prevalence in single-gene kidney diseases progressing to renal failure. Kidney Int 2000, 58:925-943.

2. Torres VE, Harris PC, Pirson Y: Autosomal dominant polycystic kidney disease. Lancet 2007, 369:1287-1301.

3. The International Polycystic Kidney Disease Consortium: Polycystic kidney disease: the complete structure of the PKD1 gene and its protein. Cell 1995, 81:289-298.

4. Mochizuki T, et al: PKD2, a gene for polycystic kidney disease that encodes an integral membrane protein. Science 1996, 272:1339-1342.

5. Hateboer N: Comparison of phenotypes of polycystic kidney disease types 1 and 2. The Lancet 1999, 353:103-107.

6. Daoust MC, Reynolds DM, Bichet DG, Somlo S: Evidence for a third genetic locus for autosomal dominant polycystic kidney disease. Genomics 1995, 25:733-736.

7. Gout AM, et al: Analysis of published PKD1 gene sequence variants. Nat Genet 2007, 39:427-8.

8. Bogdanova $\mathrm{N}$, et al: Homologues to the first gene for autosomal dominant polycystic kidney disease are pseudogenes. Genomics 2001, 74:333-341.

9. Thomas $R$, et al: Identification of mutations in the repeated part of the autosomal dominant polycystic kidney disease type 1 gene, PKD1, by long-range PCR. Am J Hum Genet 1999, 65:39-49.

10. Rossetti $\mathrm{S}$, et al: A complete mutation screen of the ADPKD genes by DHPLC. Kidney Int 2002, 61:1588-1599.

11. Rossetti $S$, et al: Comprehensive molecular diagnostics in autosomal dominant polycystic kidney disease. J Am Soc Nephrol 2007, 18:2143-60.

12. Gout AM, Martin NC, Brown AF, Ravine D: PKDB: Polycystic Kidney Disease Mutation Database-a gene variant database for autosomal dominant polycystic kidney disease. Hum Mutat 2007, 28:654-659.

13. Pei $Y$ : Diagnostic approach in autosomal dominant polycystic kidney disease. Clin J Am Soc Nephrol 2006, 1:1108-14.
14. Wittwer $C T$ : High-resolution DNA melting analysis: advancements and limitations. Hum Mutat 2009, 30:857-859.

15. Takano EA, Mitchell G, Fox SB, Dobrovic A: Rapid detection of carriers with BRCA1 and BRCA2 mutations using high resolution melting analysis. BMC Cancer 2008, 8:59.

16. Sevilla $C$, et al: Impact of gene patents on the cost-effective delivery of care: the case of BRCA1 genetic testing. Int J Technol Assess Health Care 2003, 19:287-300.

17. Pei Y, et al: Unified criteria for ultrasonographic diagnosis of ADPKD. $J$ Am Soc Nephrol 2009, 20:205-212.

18. Tan Y, et al: Novel method for genomic analysis of PKD1 and PKD2 mutations in autosomal dominant polycystic kidney disease. Hum Mutat 2009, 30:264-273.

19. Hayashi T, Mochizuki T, Reynolds DM, Wu G, Cai Y, Somlo S: Characterization of the exon structure of the polycystic kidney disease 2 gene (PKD2). Genomics 1997, 44(1):131-6.

20. Burtey S, Lossi AM, Bayle J, Berland Y, Fontés M: Mutation screening of the PKD1 transcript by RT-PCR. J Med Genet 2002, 39:422-9.

21. Ramensky V, Bork P, Sunyaev S: Human non-synonymous SNPs: server and survey. Nucleic Acids Res 2002, 30(17):3894-900.

22. Xu C, Rossetti S, Jiang L, Harris PC, Brown-Glaberman U, Wandinger-Ness A, Bacallao R, Alper SL: Human ADPKD primary cyst epithelial cells with a novel, single codon deletion in the PKD1 gene exhibit defective ciliary polycystin localization and loss of flow-induced Ca2+ signaling. Am J Physiol Renal Physiol 2007, 292:F930-945.

23. Peral B, et al: Splicing mutations of the polycystic kidney disease 1 (PKD1) gene induced by intronic deletion. Hum Mol Genet 1995, 4:569-574

24. Garcia-Gonzalez MA, et al: Evaluating the clinical utility of a molecular genetic test for polycystic kidney disease. Mol Genet Metab 2007, 92:160-167.

25. Hoefele J, Mayer K, Scholz M, Klein H-G: Novel PKD1 and PKD2 mutations in autosomal dominant polycystic kidney disease (ADPKD). Nephrol Dial Transplant 2011, 26:2181-2188.

26. Consugar $M B$, et al: Characterization of large rearrangements in autosomal dominant polycystic kidney disease and the PKD1/TSC2 contiguous gene syndrome. Kidney Int 2008, 74:1468-1479.

27. Rouleau E, et al: Quantitative PCR high-resolution melting (qPCR-HRM) curve analysis, a new approach to simultaneously screen point mutations and large rearrangements: application to MLH1 germline mutations in Lynch syndrome. Hum Mutat 2009, 30:867-875.

28. Vossen RHAM, et al: High- resolution melting analysis more than just sequence variant screening. Hum Mutat 2009, 30:861-866.

29. Harris PC, Rossetti S: Molecular diagnostics of ADPKD coming of age. Clin J Am Soc Nephrol 2008, 3:1-2

30. Huang $E$, et al: DNA testing for live kidney donors at risk for autosomal dominant polycystic kidney disease. Transplantation 2009, 87:133-137.

31. Rossetti $\mathrm{S}$, et al: Incompletely penetrant PKD1 alleles suggest a role for gene dosage in cyst initiation in polycystic kidney disease. Kidney Int 2009, 75:848-855.

32. Rossetti $\mathrm{S}$, et al: Association of mutation position in polycystic kidney disease 1 (PKD1) gene and development of a vascular phenotype. Lancet 2003, 361:2196-2201.

33. Grantham JJ, et al: Determinants of renal volume in autosomal-dominant polycystic kidney disease. Kidney Int 2007, 73:108-116.

34. Thongnoppakhun $W$, et al: Novel and de novo PKD1 mutations identified by multiple restriction fragment-single strand conformation polymorphism (MRF-SSCP). BMC Med Genet 2004, 5:2.

35. Peral $B$, et al: Screening the $3^{\prime}$ region of the polycystic kidney disease 1 (PKD1) gene reveals six novel mutations. Am J Hum Genet 1996, 58:86-96.

36. Torra R, Badenas C, Pérez-Oller L, Luis J, Millán S, Nicolau C, Oppenheimer F, Milà $M$, Darnell A: Increased prevalence of polycystic kidney disease type 2 among elderly polycystic patients. Am J Kidney Dis 2000, 36:728-34.

37. Peral $B$, et al: Identification of mutations in the duplicated region of the polycystic kidney disease 1 gene (PKD1) by a novel approach. Am J Hum Genet 1997, 60:1399-1410.

38. Torra R, et al: [Mutational analysis of the PKD1 and PKD2 (type 1 and 2 dominant autosomal polycystic kidney) genes]. Nefrologia 2000, 20:39-46.

39. Peltola $P$, et al: Genetics and phenotypic characteristics of autosomal dominant polycystic kidney disease in Finns. J Mol Med 2005, 83:638-646. 
40. Zhang $S$, et al: Mutation analysis of autosomal dominant polycystic kidney disease genes in Han Chinese. Nephron Exp Nephrol 2005, 100: e63-76.

41. Watnick T, et al: Mutation detection of PKD1 identifies a novel mutation common to three families with aneurysms and/or very-early-onset disease. Am J Hum Genet 1999, 65:1561-1571.

42. Perrichot RA, et al: DGGE screening of PKD1 gene reveals novel mutations in a large cohort of 146 unrelated patients. Hum Genet 1999, 105:231-239.

43. Rossetti S, et al: Mutation analysis of the entire PKD1 gene: genetic and diagnostic implications. Am J Hum Genet 2001, 68:46-63.

44. Phakdeekitcharoen B, Watnick TJ, Germino GG: Mutation analysis of the entire replicated portion of PKD1 using genomic DNA samples. J Am Soc Nephrol 2001, 12:955-963.

45. Afzal $A R$, et al: Novel mutations in the duplicated region of the polycystic kidney disease 1 (PKD1) gene provides supporting evidence for gene conversion. Genet Test 2000, 4:365-370.

46. Watnick TJ, et al: An unusual pattern of mutation in the duplicated portion of PKD1 is revealed by use of a novel strategy for mutation detection. Hum Mol Genet 1997, 6:1473-1481.

47. McCluskey $\mathrm{M}$, et al: Mutation detection in the duplicated region of the polycystic kidney disease 1 (PKD1) gene in PKD1-linked Australian families. Hum Mutat 2002, 19:240-250.

48. Aguiari $\mathrm{G}$, et al: Novel splicing and missense mutations in autosomal dominant polycystic kidney disease 1 (PKD1) gene: expression of mutated genes. Hum Mutat 2000, 16:444-445.

49. Rossetti $\mathrm{S}$, et al: Autosomal dominant polycystic kidney disease (ADPKD) in an Italian family carrying a novel nonsense mutation and two missense changes in exons 44 and 45 of the PKD1 Gene. Am J Med Genet 1996, 65:155-159.

50. Eo H, et al: Three novel mutations of the PKD1 gene in Korean patients with autosomal dominant polycystic kidney disease. Clin Genet 2002, 62:169-174.

51. Torra $R$, et al: Seven novel mutations of the PKD2 gene in families with autosomal dominant polycystic kidney disease. Kidney Int 1999, 56:28-33.

52. Reynolds DM, et al: Aberrant splicing in the PKD2 gene as a cause of polycystic kidney disease. J Am Soc Nephrol 1999, 10:2342-51.

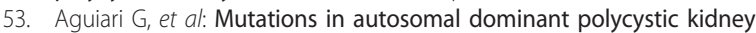
disease 2 gene: Reduced expression of PKD2 protein in lymphoblastoid cells. Am J Kidney Dis 1999, 33:880-885.

54. Badenas $C$, et al: Mutational analysis within the $3^{\prime}$ region of the PKD1 gene. Kidney Int 1999, 55:1225-1233.

\section{Pre-publication history}

The pre-publication history for this paper can be accessed here: http://www.biomedcentral.com/1471-2369/12/57/prepub

doi:10.1186/1471-2369-12-57

Cite this article as: Bataille et al: High Resolution Melt analysis for mutation screening in PKD1 and PKD2. BMC Nephrology 2011 12:57.

\section{Submit your next manuscript to BioMed Central and take full advantage of:}

- Convenient online submission

- Thorough peer review

- No space constraints or color figure charges

- Immediate publication on acceptance

- Inclusion in PubMed, CAS, Scopus and Google Scholar

- Research which is freely available for redistribution 